\title{
Gas in the life cycle of radio sources
}

\author{
Raffaella Morganti ${ }^{1}$ \\ ASTRON, the Netherlands Institute for Radio Astronomy, Postbus 2, 7990 AA, Dwingeloo, \\ The Netherlands \\ Kapteyn Astronomical Institute, University of Groningen, P.O. Box 800, 9700 AV Groningen, \\ The Netherlands \\ E-mail: morgantieastron.nI
}

Massive gas outflows are considered a key component in the process of galaxy formation and evolution. This contribution presents recent results obtained from $\mathrm{HI}$ and $\mathrm{CO}$ observations of a number of objects where the AGN - and in particular the radio jet - is playing a major role in producing massive and fast gas outflows. The results are reinforcing the conclusion that gas outflows have a complex and multiphase structure and that cold gas in different phases (atomic and molecular) represents a major component. High spatial resolution observations have allowed, in a number of sources, to locate the region where the outflow originates as well as to derive more accurate estimates of the mass outflow rate and the associated energy. Therefore, these results provide important constraints for understanding the mechanisms at the origin of the outflows.

Finally, the tendency to find of fast gas outflows in young (and/or restarted) radio sources shows how important the effect of radio plasma can be. Understanding the life cycle of radio sources is a fascinating topic that has recently gained relevance because of the impact that the radio plasma can have on its surrounding medium and, consequently, on the evolution of the host galaxy. Identify the young sources, understand their evolution, witness their effect on the surrounding medium and understand why and when a radio source stops and when restarts are all questions we hope to answer with the new generation of radio telescopes. These questions will be addressed by the combination of large surveys at low frequencies (where relics of previous activity can be more easily found) and large HI surveys to trace the presence and the characteristics of the gas in the nuclear regions. Some of the first results obtained from pilot studies in preparation to these large projects are presented here.

Resolving the Sky - Radio Interferometry: Past, Present and Future

Manchester, UK

April 17-20, 2012

1 Speaker 


\section{Outflows, feedback and galaxy evolution}

The complex interplay between different constituents of galaxies has proven to be a major stumbling block for the success of models of structure formation. For example, the growth and evolution of a galaxy is mostly determined by the balance between what and how a galaxy accretes and what expels (Silk \& Rees 1998, di Matteo et al. 2005, Hopkins et al. 2005). Accretion triggers the formation of new stars and the growth of the central massive black hole. In return, however, the energy released by star formation and nuclear activity (AGN) has strong effects on the gaseous medium in and around a galaxy. This activity can create massive outflows of gas with the effect of quenching the formation of stars and the central activity. This mechanism of energy feedback has now been recognized as key to successfully modelling structure formation. In the case of early-type galaxies, feedback is mainly provided by the energy released by the active nucleus and gas outflows are the main signature of this type of feedback in action. Studying outflows and understanding their characteristics and impact are crucial for providing key input and constraints for theoretical models (Fabian 2012).

Gaseous outflows of ionised gas have been long known to be present in AGN but their spatial extent and mass outflow rates are thought not to be comparable to what is required by feedback in numerical simulations (see e.g. Holt et al. 2011 and refs therein). However, in the last few years, the steady improvement of radio and $\mathrm{mm}$ telescopes has provided some new exciting results for our understanding of the impact of gas outflows and related feedback. Fast and massive outflows of cold (atomic and molecular) gas, as traced by the HI-21 cm and CO lines, have been detected in a growing number of objects and cold gas is now recognized as likely the most prominent phase in the outflows in terms of mass and mass outflow rate.

\section{Outflows of $\mathrm{HI}$ and molecular gas}

The discovery and study of fast and massive outflows of atomic hydrogen (HI) in radio galaxies has shown for the first time the relevance of the cold component compared to the gas locked in the warm and hot phase. Fast (up to $2000 \mathrm{~km} \mathrm{~s}^{-1}$ ) and massive (mass outflow rates up to $50 \mathrm{M}_{\odot} \mathrm{yr}^{-1}$ ) outflows of $\mathrm{HI}$ have now been found in a number of radio galaxies (Morganti et al. 1998, 2005a,b and Morganti et al. 2010 for a summary). The key role of the cold component of the gas has been recently confirmed by the detection of massive, AGN-driven outflows of molecular gas. The first case has been found in the ULIRG Mrk231 (Feruglio et al. 2010) while other interesting examples have been found in NGC1266 by Alatalo et al. (2011), and in 4C12.50 by Dasyra \& Combes (2012). Although limited for now to a handful of objects, these results suggest the idea that $A G N$-driven outflows have a complex and multiphase structure where cold gas in different phases is involved and possibly represents the major component.

Mrk231 and NGC1266 are at the opposite side of the distribution of objects where cold gas outflows have been found. The recent detection of molecular gas outflow in the Ultra-Luminous advanced major merger Mrk231 (Feruglio et al. 2010) has triggered observations also in HI and the result is shown in Morganti et al. (2011). In addition to the already known HI absorption feature centered on the systemic velocity (and attributed to a circumnuclear disk), we find a 
blueshifted wing, albeit with a much smaller amplitude than the $\mathrm{CO}$ one. This indicates that the molecular outflow has associated also a component of neutral atomic gas. Although Mrk231 is a radio source with a complex structure, the role of the radio plasma (if any) in producing the outflow in this object is not clear and it will have to be investigated with observations with higher spatial resolution.

At the opposite extreme is the case of the early-type galaxy NGC1266 (Alatalo et al. 2011) showing that outflows of cold gas can also occur in relatively quiet early-type galaxies. This object hosts a weak radio source $\left(\sim 10^{21} \mathrm{~W} / \mathrm{Hz}\right)$ and single-dish and interferometric observations have found a massive, centrally concentrated molecular component with a $\mathrm{H}_{2}$ mass of $1.1 \times 10^{9}$ $\mathrm{M}_{\odot}$ and a molecular outflow with a mass of $\sim 2.4 \times 10^{7} \mathrm{M}_{\odot} \mathrm{yr}^{-1}$. The star formation in NGC 1266 is insufficient to drive the outflow, and thus it is likely driven by the active galactic nucleus. The role of radio plasma in this object is under investigation with higher resolution radio observations. The estimated mass outflow rate of $\sim 13 \mathrm{M}_{\odot} \mathrm{yr}^{-1}$ leads to a depletion timescale (of the circumnuclear gas) of $\sim 85 \mathrm{Myr}$. This relatively short time may explain why the phenomenon of gaseous outflow is rare in early-type galaxies (Alatalo et al. 2011). Apart from these two objects, a number of galaxies where outflows of cold gas (atomic and/or molecular) have been observed so far are radio-loud AGN. Evidence that the radio plasma plays an important role in originating the outflows in these objects has been found in a number of clear cases (e.g. Morganti et al. 2005a,b; Oosterloo et al. 2000; Dasyra \& Combes 2012).

Here we present the results from some specific objects studied so far and we describe the characteristics of the outflows in their central regions (up to few $\mathrm{kpc}$ ). Apart from being interesting for feedback and galaxy evolution, the effects of interaction between radio plasma and ISM are also relevant for the evolution and life of the radio source itself.

\section{The intriguing rejuvenating radio sources}

An intriguing relation between the presence of outflows and the stage in the life of the radio source has been suggested by the tendency to find $\mathrm{HI}$ and molecular gas outflows in young or recently restarted radio AGN (i.e. where a low-surface brightness extended component of radio emission from the previous radio-loud phase), see Morganti et al. 2005a. Unfortunately the statistics is now very limited to full confirm this trend. Some of these objects used to be the favourite objects of Richard Schilizzi when he was still an active astronomer! For example, the famous double-double and recently restarted radio source 3C236 (Schilizzi et al. 2001, Tremblay et al. 2010) where we have now confirmed the presence of the fast outflow of HI (presented for the first time in Morganti et al. 2005a) using new EVLA observations.

This trend is consistent with the more general tendency of young/restarted radio sources to be more likely detect in HI, i.e. to be more likely rich in gas in their nuclear regions (see e.g. Saikia et al. 2007).

If this trend is confirmed once larger samples will be available, this would be extremely interesting because it would connect the presence of outflows with the evolutionary stage of the radio source, consistent with what expected by theoretical models of feedback. 
An interesting example of these types of objects where recently the molecular counterpart of the HI outflow has been observed (see Dasyra \& Combes 2012) is the Gigahertz-Peaked source (GPS) and far-IR bright $4 \mathrm{C} 12.50$, hosted in an on-going merger (see Fig. 1). This source also shows radio emission on the kpc scale (Stanghellini et al. 2005) suggesting that the radio-loud phase is very recently restarted. The HI appears to be located in clouds, indicating that the very distorted radio jets (Lister et al. 2004) are expanding in a hostile, clumpy medium fighting their way out (Morganti et al. 2004). This is similar to what numerical simulations predict for these young radio sources in their first phase of their evolution (Wagner \& Bicknell 2011, 2012). The simulations also suggest that the effect of the interaction between radio jet and ISM extend to large distance from the jet, heavily perturbing and shocking the ISM in the galaxy.

\section{Radio jets as driver of outflows}

Outflows can be driven by different

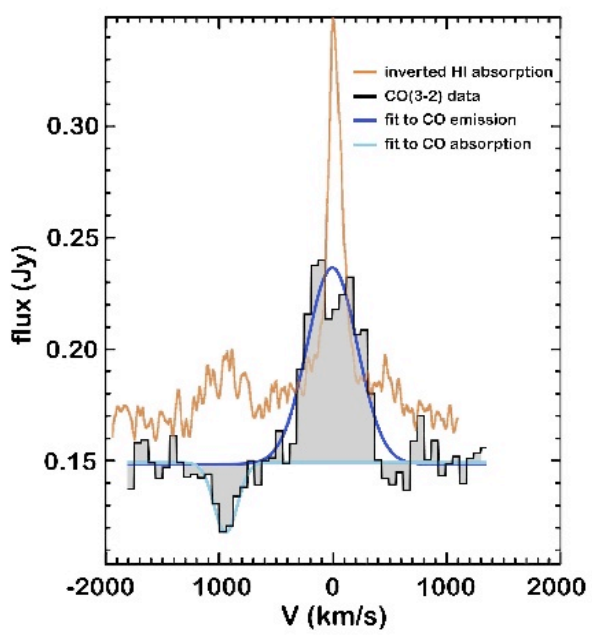

Figure $1-{ }^{12} \mathrm{CO}(3-2)$, profile from Dasyra \& Combes (2012) showing the emission and the absorption component at $-950 \mathrm{~km} \mathrm{~s}^{-1}$ (grey filled profile). The inverse $H I$ absorption that is seen in the radio data of 4C12.50 (Morganti et al, 2004) is overplotted in orange with mechanisms related to the active nucleus. What is an arbitrary scale and continuum the dominant mechanism, e.g. radiation pressure $v s$ mechanical action from radio jets, has implications on what type of nuclear activity has the highest impact on galaxy evolution. Radio emission and, in particular, plasma jets, are one of the main candidates for driving gaseous outflows (see e.g. Croton et al. 2006 and the numerical simulations e.g. Wagner \& Bicknell 2011, 2012; Gaibler et al. 2011). Radio has given major input on this issue, for example through the study of radio lobes and X-ray bubbles (McNamara \& Nulsen 2012). Indeed, radio jets provide a particularly suitable and fast way of injecting energy into the large-scale surrounding medium because they couple efficiently to the ISM/IGM. But the effects of the radio plasma actually start in the central regions. The discovery that in most of the HI cases available so far for radio-loud AGN, the outflow is occurring at hundred of pc to kpc distances from the nucleus and at the location of bright radio features (see e.g. Morganti et al. 2005a,b), strongly suggests that the fast $\mathrm{HI}$ outflows are driven by the interactions between the expanding radio jets and the gaseous medium enshrouding the central regions and emphasis the important of radio-loud AGN for feedback.

On theoretical ground, it may be even expected that most of the mass associated with such outflows is colder and molecular (Mellema et al. 2004; Fragile et al. 2004, Zubovas \& King 2012, Narayanan et al. 2008) and that the effects of this component on galaxy evolution may be large. Two possible scenarios can be envisaged to explain the origin of these outflows. The preexisting molecular gas is entrained and accelerated by the cocoon around the radio jets/lobes or, alternatively, the molecular gas is formed out of (efficiently) cooling outflowing atomic gas. 
After the gas is shocked by the passage of the jet, radiative cooling can be very efficient and the gas quickly cools to very low temperatures. In this scenario, HI would possibly represent an intermediate phase in the cooling process, while the molecular gas would be the final stage.

Progress in the study of the amplitude and the impact of feedback effects requires to expand the number of observed objects to be able to build statistics and relate the presence and the characteristics of the outflow to the type of AGN. However, it is also important to perform detailed studies of single objects to explore the physical conditions, distribution and kinematics of the gas and the impact of the outflows on the galaxy (e.g. by comparing to the Eddington and bolometric luminosity, to the star formation rate etc.).

\section{A show case of jet/ISM interaction: the radio-loud Seyfert galaxy IC5063.}

The radio-loud Seyfert 2 galaxy IC5063 was the first object where a fast HI outflow was detected (Morganti et al. 1998) and localised (Oosterloo et al. 2000), roughly $0.5 \mathrm{kpc}$ (about 2")

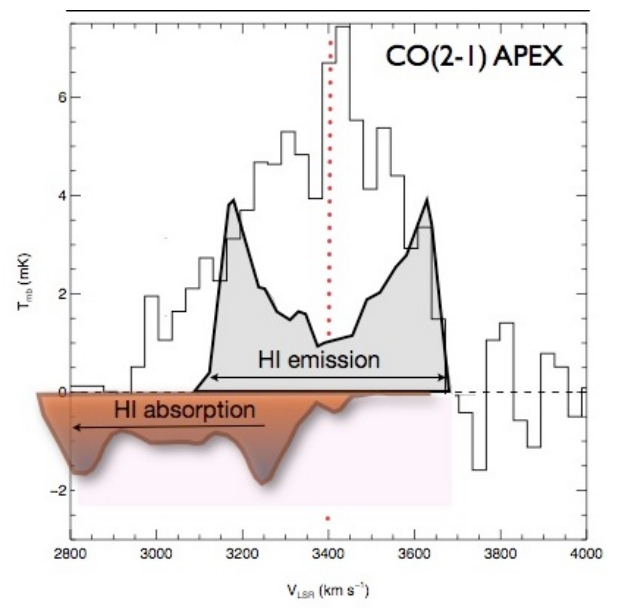

Figure 2 - Profile obtained from the APEX CO (2-1) observations of IC5063. The profile is extremely broad (almost 800 $\mathrm{km} / \mathrm{s} \mathrm{FWZI}$ ) and asymmetric showing a pronounced blueshifted "wing". The profile reaches velocities well outside the range covered by the regularly rotating large-scale gas disk observed in HI. The bluewards "wing" of the CO(2-1) profile covers velocities similar to those of the HI outflow detected in absorption (Morganti et al, in prep). this assumption about the location, we can derive
some parameters for the molecular outflow. The $\mathrm{H}_{2}$ mass involved in this outflow is between $8 \times 10^{6}$ and $4 \times 10^{7} \mathrm{M}_{\odot}$, making this component, as in other cases, more massive compared to the $\mathrm{HI}$ or the ionised gas components. The mass outflow rate is estimated between 8 and $40 \mathrm{M}_{\odot} \mathrm{yr}^{-1}$, implying a depletion time of at least $10 \mathrm{Myr}$. These values are comparable to what found for some of the other objects where outflows of molecular gas have been detected, with the exception of the most extreme case of Mrk 231. offset from the nucleus at the location of the bright $\mathrm{W}$ radio lobe. At the same location, a fast - but less massive - outflow of warm, ionised gas has also been found (Morganti et al. 2007). Recent Atacama Pathfinder EXperiment (APEX) observations show that the observed $\mathrm{CO}(2-1)$ profile is asymmetric with a blueshifted "wing" that extends to much larger velocities compared to those covered by the regular rotation of the large-scale gas disk (of which the velocities are known from the HI observations). Figure 2 illustrates the comparison between molecular $\mathrm{CO}(2-1)$ and $\mathrm{HI}$ integrated profiles. This clearly indicates the presence of a molecular component in the outflow in this radio source. Given the similarities between $\mathrm{HI}$ and $\mathrm{CO}$ profiles, the outflow of molecular gas could be located at the position of the western radio lobe, i.e. about $0.5 \mathrm{kpc}$ from the nucleus, as in the case of the HI. If this is confirmed by higher resolution observations, it will represent the first case of jet-cloud interaction producing also a molecular outflow. Keeping in mind 


\section{The importance of locating the outflow: $3 \mathrm{C293}$}

Identify the location of the outflows is essential in order to understand their origin and derive important parameters (e.g. mass, mass outflow rate etc.). As mentioned above (Sec. 4), we know a number of cases where the outflow is occurring at hundred of pc to kpc distances from the nucleus and at the location of a bright radio-jet hot-spots (see e.g. Oosterloo et al. 2000, Morganti et al. 2005b), strongly suggesting that the fast HI outflows are often driven by the interactions between the expanding radio jets and the gaseous medium.

Using EVLA observations, we have now a new example of this in the nearby radio galaxy 3C293. In this object we have found that the outflow is coming form the inner radio lobe (see Fig. 3 for details). From this outflow we derive a mass outflow rate of $\mathrm{M}_{\odot} \sim 50 \mathrm{M}_{\odot} / \mathrm{yr}$, comparable (although at the lower end of the scale) to the supernovae driven outflows in ULIRGS. Given the relatively low star formation rate of this galaxy $\left(\sim 4 \mathrm{M}_{\odot} / \mathrm{yr}\right.$, Papadopoulos et
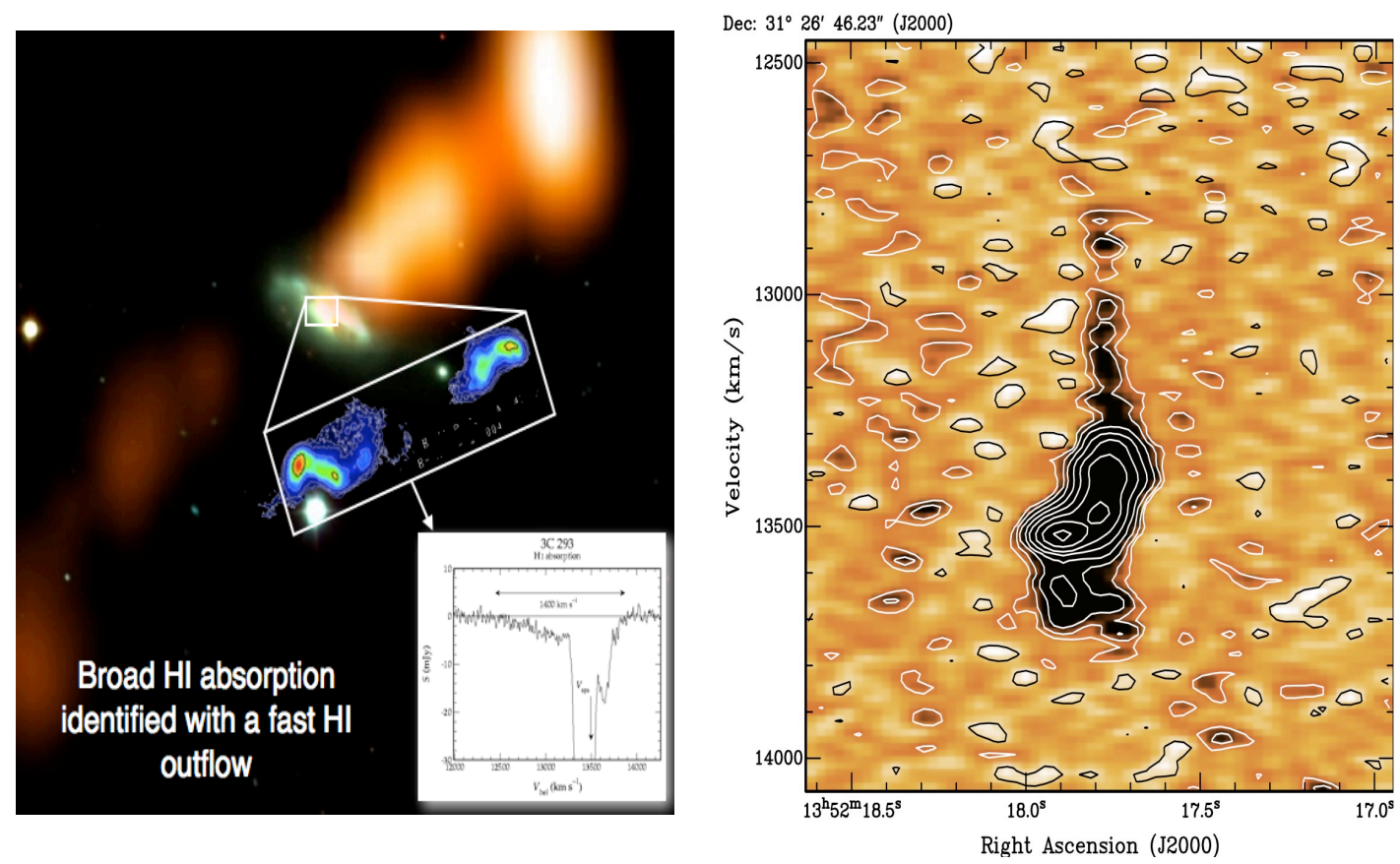

Figure 3 - Right - Radio continuum of the large-scale (orange, Morganti et al. 2003) and small restarted part (blue, Beswick et al. 2004) of the radio galaxy $3 C 293$ overlaid to the optical image of the host galaxy. The inset shows the extremely broad $(>1000 \mathrm{~km} / \mathrm{s}) \mathrm{HI}$ profile detected against the inner part (Morganti et al. 2003). Left-Position-velocity plot illustrating the location of the blueshifted) absorption on the $W$ lobe (Mahony et al, in prep).

al. 2010), this outflow is clearly sufficient to quench star formation in the host galaxy. This is similar to the outflows of CO observed in nearby LIRGS/ULIRGS (e.g. Feruglio et al., 2010), although the outflow rate is not as large. However, this implies that outflows are present over a large amount of the AGN's lifetime, not only in the early ULIRG stages. It is also consistent with the high excitation CO lines observed in 3C293, believed to be induced by shocks created by this jet-ISM interaction (Papadopoulos et al. 2008, 2010). 


\section{Where are we now and where we want to go}

The main results from our studies so far can be summarized in: $i$ ) outflow of atomic and molecular gas are found in a growing number of radio sources; ii) these outflows are complex and multiphase structures; iii) the location of the outflow is now better known in a number of cases; $i v$ ) the cold gas appears to be the dominant component in jet-induced outflows despite the high energies dumped in the ISM by radio jets.

From the description above it is clear that two essential ingredients are needed to make progress in this topic. The first is to quantify the relevance of outflows for galaxy evolution by having a better statistics on the occurrence of outflows of cold gas in radio galaxies. For this, more sensitive HI observations are needed. The second is to quantify the occurrence of a radio-loud phase in the life of a galaxy and explore the apparent connection between this cycle and the presence of gas. For this, we need blind, deep search for radio relic structures around galaxies and link them to the presence of gas in these objects.

A possibility to go beyond the limitations of current HI surveys is given by stacking analysis, allowing one to statistically investigate the global HI properties of galaxies at higher redshifts. (Lah et al. 2009, Fabello et al. 2011, Gereb et al. 2012). With the advent of broadband capabilities of the radio telescopes in combination with relatively high spectral resolution (now relatively common even for continuum observations), stacking techniques can be applied to almost any available datasets. This gives the possibility to reach, even now, microJy level sensitivity in HI. A pilot stacking analysis of galaxies in the Lockman Hole area has been carried out to demonstrate that stacking is
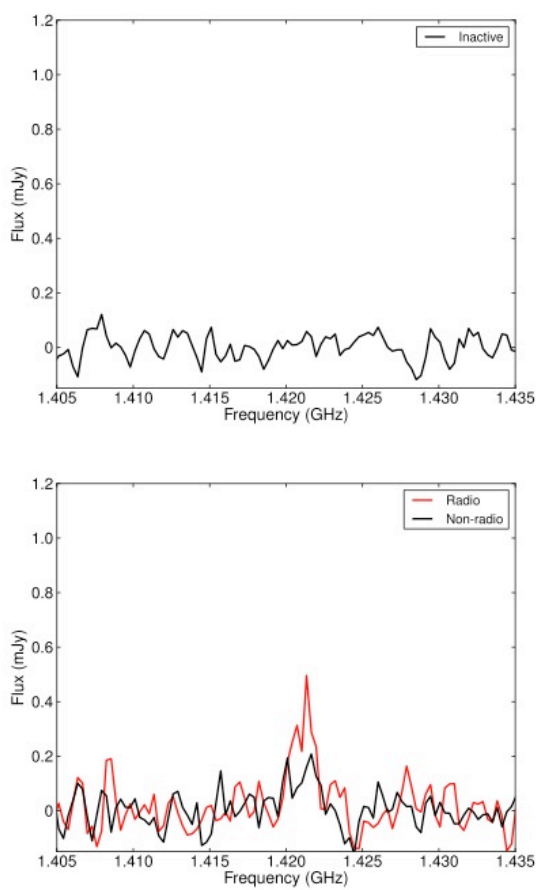

Figure 4 - Stacked profiles of red inactive galaxies (above) and LINERs (below) as derived from the Lockman Hole field (see Gereb et al. 2012 for details). This illustrates the increased sensitivity to microJy level and the possibility of easily detecting HI even in red galaxy at higher redshift than usually possible (up to 0.09 in this study). an effective technique for global HI studies. This area allowed us to investigate, for the first time, both continuum (Guglielmino et al. 2012) and line (Gereb et al. 2012) properties of galaxies in the field. Figure 3 shows an example of HI detection for radio detected sub-mJy sources (see Gereb et al. 2012 for details). In case of stacking, an extra advantage is provided by the weaker requirements for calibration and continuum subtraction, making it easier to reduce and analyze data with automated pipelines in the future. Exploring the possibility of using stacking also to detect $\mathrm{HI}$ absorption is in progress. 
The possibility of learning more about the life-cycle of radio sources is better done by observations at low frequencies (taking advantage of the steep spectral index of relic structures) over wide field of view (as relic structures are known to be rare). This is now offered by LOFAR (van Haarlem et al. 2012) and observations to tackle this have already started. The success of this search will depend on the full sensitivity and the multi-frequencies capability of this instrument that is now in its commissioning phase but the first results are already extremely encouraging.

The results presented here would not have been obtained without the help of my collaborators. In particular I would like to thank Clive Tadhunter, Tom Oosterloo, Raymond Oonk, Wilfred Frieswijk, Katinka Gereb, Elizabeth Mahony, Katey Alatalo and the ATLAS3D collaboration.

\section{References}

[1] Alatalo et al. 2011 ApJ 735, 88;

[2] Croton et al. 2006 MNRAS 365, 11

[3] Dasyra K.M., Combes F., 2012, A\&A, 541, L7

[4] Di Matteo et al. 2005, Nature 433, 604;

[5] Fabello S., Kauffmann G., Catinella B. et al., 2011, MNRAS, 416, 1739

[6] Fabian, A. 2012, ARAA (arXiv:1204.4114)

[7] Feruglio et al. 2010, A\&A Letter in press (astro-ph/1006.1655);

[8] Fragile, P. C., Murray, S. D., Anninos, P., van Breugel, W. 2004, ApJ 604, 74

[9] Gaibler V., Khochfar S., Krause M., Silk J., 2011 (arXiv:1111.4478)

[10] Gereb K., Oosterloo T., Morganti R., Prandoni I., Guglielmino G. 2012, A\&A submitted

[11] Guglielmino G., Prandoni I., Morganti R. 2012 Proceedings of the meeting "Resolving The Sky - Radio Interferometry: Past, Present and Future", PoS(RTS2012)022

[12] Holt J.; Tadhunter, C. N.; Morganti, R.; Emonts, B. H. C. 2011, MNRAS 410, 1527

[13] Hopkins P. F., Hernquist L., Cox T. J., et al. 2005, ApJ, 630, 705

[14] Lah P. et al. 2009, MNRAS, 399, 1447

[15] McNamara B.R., Nulsen P.E.J., 2012, NJPh, 14, 055023

[16] Mellema G., Kurk \& Rottgering H. 2002, A\&A 395L, 13

[17] Morganti, R., Tadhunter, C. N., Oosterloo, T. A. 2005a A\&A 444, L9;

[18] Morganti R., Oosterloo T.A., Tadhunter C.N., van Moorsel G., Emonts B., 2005b, A\&A, 439, 521 
[19] Morganti R., Holt J., Tadhunter C., Oosterloo T., 2010, “Co-Evolution of Central Black Holes and Galaxies" IAUS, 267, 429 2010IAUS..267..429;

[20] Morganti et al. 2011 Proceedings of the 11th Asian-Pacific Regional IAU Meeting; NARIT Conference Series, Vol. 1, S. Komonjinda, Y. Kovalev, and D. Ruffolo, eds. 2011arXiv1112.5093

[21] Morganti et al. 2007 A\&A 476, 735;

[22] Morganti R., Oosterloo T.A., Tadhunter C.N., Vermeulen R., Pihlstrom Y.M., van Moorsel G., Wills K.A., 2004, A\&A, 424, 119

[23] Morganti et al. 1998 AJ, 115, 915;

[24] Narayanan, D., Cox, T. J., Kelly, B., et al. 2008, ApJS, 176, 331

[25] Oosterloo et al. 2000, AJ, 119, 2085;

[26] Papadopoulos et al., 2008, A\&A 491, 483;

[27] Papadopoulos et al., 2010, ApJ 715, 775;

[28] Schilizzi R.T., et al., 2001, A\&A, 368, 398

[29] Saikia, D.J., Gupta, N. \& Konar, C., 2007, MNRAS, 375, L31

[30] Silk \& Rees, 1998, A\&A, 331, L1

[31] Stanghellini C., O'Dea C.P., Dallacasa D., Cassaro P., Baum S.A., Fanti R., Fanti C., 2005, A\&A, 443, 891

[32] Tremblay G.R., O'Dea C.P., Baum S.A., Koekemoer A.M., Sparks W.B., de Bruyn G., Schoenmakers A.P., 2010, ApJ, 715, 172

[33] van Haarlem et al. 2012 A\&A submitted

[34] Zubovas K., King A., 2012, ApJ, 745, L34

[35] Wagner \& Bicknell, 2011, ApJ 728, 29;

[36] Wagner A.Y., Bicknell G.V., Umemura M., 2012, arXiv, arXiv:1205.0542 